\title{
Entropy Production Rate for Avascular Tumor Growth
}

\author{
Elena Izquierdo-Kulich, Esther Alonso-Becerra, José M. Nieto-Villar \\ Department of Physical Chemistry, Ave. Zapata \& G, Faculty of Chemistry, University of Havana, Havana, Cuba \\ E-mail: nieto@fq.uh.cu \\ Received January 17, 2011; revised March 26, 2011; accepted April 1, 2011
}

\begin{abstract}
The entropy production rate was determined for avascular tumor growth. The proposed formula relates the fractal dimension of the tumor contour with the quotient between mitosis and apoptosis rate, which can be used to characterize the degree of proliferation of tumor cells. The entropy production rate was determined for fourteen tumor cell lines as a physical function of cancer robustness. The entropy production rate is a hallmark that allows us the possibility of prognosis of tumor proliferation and invasion capacities, key factors to improve cancer therapy.
\end{abstract}

Keywords: Entropy Production Rate, Cancer Robustness, Cancer Evolution

\section{Introduction}

Cancer is a generic name given to a group of malignant cells which have lost their specialization and control over normal growth. These groups of malignant cells are nonlinear dynamic systems which self-organize in time and space, far from thermodynamic equilibrium, and exhibit high complexity [1], robustness [2] and adaptability [3].

In spite of achievements in molecular biology and genomics, the growth mechanism for tumor cells and the nature of its robustness are still unknown. According to Kitano [4,5], cancer robustness is due to functional redundancy and feed-back control systems. This robustness enables a system to maintain its functionality in the face of various external and internal perturbations.

For Hauptmann [6], cancer is an adaptive phenomenon which is the response to cellular stress induced by an energetic overload which ultimately leads to an increase in cellular entropy. Recently Luo [7] demonstrated that the entropy production rate of cancer cells is always higher than that of healthy cells.

In previous work it was demonstrated that the entropy production rate is a Lyapunov function [8]. The objective of this work is to extend the thermodynamic formalism as applied to cancer, leading us to the suggestion that the entropy production rate for avascular tumor growth is a "hallmark of cancer". The plan of the paper is the following: Section 2 gives a summary of the phenomenology of irreversible processes and sets the stage for the results of entropy production rate to follow. In Section 3, a formalism is obtained from the master equation (ME) to obtain the mesoscopic model which describes the tumor growth dynamics in absence of external fluctuations, taking into account that the tumor grows in a limited area. The microscopic variable considered to describe the state of the system is the total number of tumor cells, and the macroscopic variables are the expected value of the radius and the fractal dimension, which is a result of internal fluctuations. In Section 4, Results and Discussion, the behavior of different types of tumor cell colonies, characterized by Brú [9] is predicted by using the formalism developed in Section 2 and 3; and finally, some conclusions are presented.

\section{Thermodynamic Formalism}

We know from classic thermodynamics that if the constraints of a system are the temperature $T$ and the pressure $P$; the entropy production can be evaluated using Gibbs's free energy [10], as:

$$
\delta S_{i}=-\frac{1}{T} \mathrm{~d} G_{T P}
$$

If the time derivative of (1) is taken, we have that:

$$
\frac{\delta S_{i}}{\mathrm{~d} t}=-\frac{1}{T} \frac{\mathrm{d} G_{T P}}{\mathrm{~d} t}
$$

where $\delta S_{i} / \mathrm{d} t$ represents the entropy production rate, $\dot{S}_{i}$. The term $\mathrm{dG}_{T P} / \mathrm{d} t$ can be developed by means of the chain rule as a function of the degree of advance of the reaction as: 


$$
\frac{\mathrm{d} G_{T P}}{\mathrm{~d} t}=\left(\frac{\partial G}{\partial \xi}\right)_{T P} \frac{\mathrm{d} \xi}{\mathrm{d} t}
$$

where $(\partial G / \partial \xi)_{T P}$ - according to De Donder and Van Rysselberghe [11] — represents the affinity $A$, with opposed sign, and the term $\mathrm{d} \xi / \mathrm{d} t$ is the reaction rate $\dot{\xi}$. Taking into account (2) and (3), we get:

$$
\frac{\delta S_{i}}{\mathrm{~d} t}=\dot{S}_{i}=\frac{1}{T} A \dot{\xi}
$$

The affinity can be calculated as [9]:

$$
\mathrm{A}=R T \ln \left(\frac{K_{C}}{\prod C_{k}^{v(k)}}\right)
$$

where $K_{c}=k_{f} / k_{b}$ is the Guldberg-Waage constant ( $k_{f}$ and $k_{b}$ are the forward and backward rate constants), $C_{k}$ is the concentration of the species $k$, whose stoichiometric coefficients $v(k)$ are negative for reactants and positive for a products. The formula (5) we rewrite as:

$$
\mathrm{A}=R T \ln \left(\frac{k_{f} \prod C_{k(f)}^{v(k)}}{k_{b} \prod C_{k(b)}^{v(k)}}\right)
$$

The reaction rate $\dot{\xi}$ can be evaluated according to the difference between the forward $\dot{\xi}_{f}$ and backward reaction rates $\dot{\xi}_{b}$, as:

$$
\dot{\xi}=\left(\dot{\xi}_{f}-\dot{\xi}_{b}\right)=k_{f} \prod C_{k(f)}^{v(k)}-k_{b} \prod C_{k(b)}^{v(k)}
$$

Substituting (6) and (5a) on (4) is obtained:

$$
\dot{S}_{i}=R\left(\dot{\xi}_{f}-\dot{\xi}_{b}\right) \ln \frac{\dot{\xi}_{f}}{\dot{\xi}_{b}} \geq 0
$$

The formula (7) is always positive by virtue of the second law. As demonstrated as a proof in reference [8] the relation (7) is a Lyapunov function, and thus provides a directional criterion and stability for the dynamical system, in other words, characterizes a complexity of the system. As a matter of fact, we postulate the entropy production given by (7) as a "hallmark of cancer" useful to the prognosis of tumor proliferation.

\section{Mesoscopic Model}

To obtain a mathematical model to predict avascular tumor growth, the following considerations were made:

First: The considered system is a tumor in vitro with a circular geometry in 2D and an irregular contour, where the increase of the number of cells $n$ occurs because of the reproduction of the contour cells. The total number of cells $n$ is the microscopic variable that describes the behavior of the system, and macroscopic variables considered were the tumor radius $r$ and the fractal dimension of the interface $d_{f}$, related by the expression:

$$
\begin{gathered}
n=\frac{\pi r^{2}}{\Omega}, \\
d_{f}=2-\frac{1}{2} G(y), \\
y=\lim _{l \rightarrow 1} \frac{\Delta \ln (w)}{\Delta \ln (l)},
\end{gathered}
$$

where $\Omega\left[L^{2}\right]$ is the area occupied by an individual cell, $w$ is an non-dimensional magnitude that express the height difference between two points in the contour separated by an non-dimensional distance $l$, and $G(y)$ is a linear function of $y$. Because the reproduction and death of the cells on the contour are considered as stochastic process, $r$ is a stochastic variable who's variance is related with the contour roughness.

Geometrically, a tumor has the shape shown in Figure $\mathbf{1}$, in which the distance between the centre of the tumor and the point at the interface more distant from the centre $H[L]$, the expected value of the tumor radius $R[L]$, and the difference between the maximum heights of two points in the contour $W[L]$ are useful variables. (What is the variable L?)

Second: As the contour rugosity is a property of the tumour, not all the surface of radius $H$ is covered by tumour cells. If it is considered that internal fluctuations scale with the area occupied by the microscopic entities that characterize the tumour (tumour cells) then the percentage of the host area occupied by tumour cells depends on the relation between the size of the entity and the expected value of the area occupied by the tumour, expressed by:

$$
\frac{R^{2}}{H^{2}}=f\left(\frac{\Omega}{R^{2}}\right),
$$

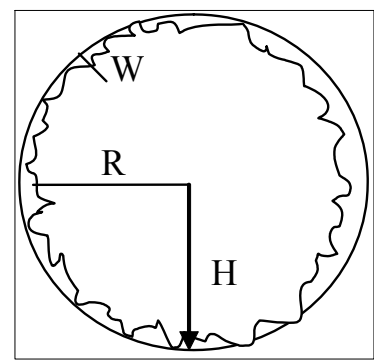

Figure 1. Geometry representation of the tumour: $H[L]$ is the distance between the centre of the tumour and the point at the interface most distant from the centre, $R[L]$ is the expected value of the tumour radius and $W[L]$ the difference between the maximum heights of two points on the contour. 
where $f$ is a function of the relation $\left(\Omega / R^{2}\right)$ with the following properties:

I think the function $f$ is missing in these following 2 equations??

$$
\lim _{\frac{\Omega}{R^{2}} \rightarrow o}\left(\frac{\Omega}{R^{2}}\right)=1 \Rightarrow W=0 ; d_{f}=1
$$

and

$$
\lim _{\frac{\Omega}{R^{2}} \rightarrow \infty}\left(\frac{\Omega}{R^{2}}\right)=0 \Rightarrow W=\infty ; d_{f}=2
$$

Third: Because the change of $n$ depends of the proliferation and death of the contour cells and if the formula (7) is considered, then the transition probability per unit of time $T_{r}\left[t^{-1}\right]$ associated with the increases of $n$ is written a priori as:

$$
T_{r}=\mu n^{0.5}
$$

While that the transition probability per unit of time associated to the decrease of $n, T_{d}\left[t^{-1}\right]$ is assumed as:

$$
T_{d}=k_{d} n^{0.5}
$$

where:

$$
\begin{aligned}
& k_{d}=b\left(1+F_{a}\right) ; \text { What is ctc?? } \\
& b=c t c ; \\
& F_{a}=\frac{r^{2}}{D^{2}}=\frac{n}{N} .
\end{aligned}
$$

In Equations (14) and (15) $\mu\left[t^{-1}\right]$ is the cell reproduction rate constant, and $k_{d}\left[t^{-1}\right]$ is the cell death rate constant. The death rate constant $k_{d}$ includes a correction term $F_{a}$, which represents the relation between the tumour radius $r$ and a characteristic length $D$ of the area (see Equations (16) and (17)) and takes into account the finite area of the host. The term $F_{a}$ is equivalent to the relation between the total number of cells and the total sites which can be occupied.

Considering the transition probabilities (14) and (15) the master equation ME [12] which describes the probability behaviour $P(n ; t)$ of having $n$ cells in time $t$ is written as:

$$
\begin{aligned}
\frac{\partial P(n ; t)}{\partial t}= & \left(\mathrm{E}_{n}^{-1}-1\right) \mu n^{0.5} P(n ; t) \\
& +\left(\mathrm{E}_{n}^{+1}-1\right) b\left(1+\frac{n}{N}\right) n^{0.5} P(n ; t), \\
P\left(n_{0} ; 0\right)= & 1,
\end{aligned}
$$

where $\mathrm{E}_{n}^{a}$ is the step operator.

Since the reproduction or death of a single cell produces a negligible effect on the system:

$$
\frac{\Delta n}{n} \approx 0,
$$

then the variable $n$ can be considered continuous. If the step operator is expressed in its differential form:

$$
\begin{aligned}
& \mathrm{E}_{n}^{+1}=1+\frac{\partial}{\partial n}+\frac{1}{2} \frac{\partial^{2}}{\partial n^{2}}, \\
& \mathrm{E}_{n}^{-1}=1-\frac{\partial}{\partial n}+\frac{1}{2} \frac{\partial^{2}}{\partial n^{2}},
\end{aligned}
$$

The Fokker-Planck equation (FPE) is obtained $[12,13]$ for $P(n, t)$ :

$$
\begin{aligned}
\frac{\partial P(n ; t)}{\partial t}= & -\frac{\partial}{\partial n}\left[\mu n^{0.5}-b\left(1+\frac{n}{N}\right) n^{0.5} P(n ; t)\right] \\
& +\frac{1}{2} \frac{\partial^{2}}{\partial n^{2}}\left[\left(\mu n^{0.5}+b\left(1+\frac{n}{N}\right) n^{0.5}\right) P(n ; t)\right]
\end{aligned}
$$

If we take into account the following relations between the probability related to the microscopic $P(n, t)$ and the one related to the macroscopic variables $P(r, t)$ [13]:

$$
\begin{gathered}
P(n ; t) \partial n=P(r ; t) \partial r ; \\
\frac{\partial P(n ; t)}{\partial t}=\frac{\partial r}{\partial n} \frac{\partial P(r ; t)}{\partial t},
\end{gathered}
$$

then the FPE related to the behavior of the macroscopic variable is:

$$
\begin{aligned}
\frac{\partial P(r ; t)}{\partial t}= & -\frac{\partial}{\partial r}\left[\left(\psi-\eta\left(1+\frac{r^{2}}{D^{2}}\right)-\frac{\Omega}{2 r^{2}}\left(\psi+\eta\left(1+\frac{r^{2}}{D^{2}}\right)\right)\right) P(r ; t)\right] \\
& +\frac{1}{2} \frac{\partial^{2}}{\partial r^{2}}\left[\frac{\Omega}{2 r}\left(\psi+\eta\left(1+\frac{r^{2}}{D^{2}}\right)\right) P(r ; t)\right],
\end{aligned}
$$

in which the relations among macroscopic and microscopic rate constants are:

$$
\begin{aligned}
& \psi=\left(\frac{\Omega}{4}\right)^{0.5} \mu \\
& \eta=\left(\frac{\Omega}{4}\right)^{0.5} b .
\end{aligned}
$$

In FPE (XXV), the first term on the right is a convective term related to the expected or deterministic value, while the second term is a diffusive term related to the fluctuations value. Taking into account that the macroscopically observed cell size $\Omega$ is independent of the tumour size $r^{2}$, we can consider that:

$$
\psi-\eta\left(1+\frac{r^{2}}{D^{2}}\right) \gg \frac{\Omega}{2 r^{2}}\left(\psi+\eta\left(1+\frac{r^{2}}{D^{2}}\right)\right)
$$


in such a way that Equation (25) is written as:

$$
\begin{aligned}
\frac{\partial P(r ; t)}{\partial t}= & -\frac{\partial}{\partial r}\left[\left(\psi-\eta\left(1+\frac{r^{2}}{D^{2}}\right)\right) P(r ; t)\right] \\
& +\frac{1}{2} \frac{\partial^{2}}{\partial r^{2}}\left[\frac{\Omega}{2 r}\left(\psi+\eta\left(1+\frac{r^{2}}{D^{2}}\right)\right) P(r ; t)\right], \\
P\left(r_{0} ; t_{0}\right)= & 1
\end{aligned}
$$

From the FPE (29) the expected radius of the tumour $R$ is obtained [12]:

$$
\begin{aligned}
& \frac{\mathrm{d} R}{\mathrm{~d} t}=\psi-\eta\left(1+\frac{R^{2}}{D^{2}}\right) ; \quad \frac{\mathrm{d} R}{\mathrm{~d} t}=\dot{\xi}-\eta \frac{R^{2}}{D^{2}}, \\
& R_{0}>0
\end{aligned}
$$

where $\psi$ and $\eta\left[L . t^{-1}\right]$ are the macroscopic parameters associated to mitosis and apoptosis rate and $\dot{\xi}\left[L . t^{-1}\right]$ is the tumour growth rate $(\dot{\xi}=\psi-\eta)$ macroscopically observed during the linear growth stage [9]; and for variance $\sigma$.

$$
\begin{aligned}
& \frac{\mathrm{d} \sigma}{\mathrm{d} t}=-2 \frac{\eta R}{D^{2}} \sigma+\frac{\Omega}{2 R}\left(\psi+\eta\left(1+\frac{R^{2}}{D^{2}}\right)\right), \\
& \sigma_{0}>0
\end{aligned}
$$

The system of ordinary differential equations given by (30) and (31) represents the mesoscopic model which describes the tumour dynamics in absence of external fluctuations considering the finite host area.

The stability analysis [14] shows that the radius grows to a stable stationary state, also called dormant tumour stage [15].

Forth: The tumour fractal dimension depends on the physiological condition of active cells at the interface, and it must include the reproduction and death rate constants. To determine the characteristic fractal dimension of the tumour, the right side of Equation (31) is equalled to zero, so:

$$
\frac{\mathrm{d} \sigma}{\mathrm{d} t}=0 ; D=H
$$

and the variance is expressed as:

$$
\sigma=\frac{H^{2}}{4} \frac{\Omega}{R^{2}}\left(\frac{\psi}{\eta}+1+\frac{R^{2}}{H^{2}}\right) .
$$

As the height difference between two points at the interface is equivalent to the magnitude of internal fluctuations, expressed by the square root of the variance [16], the following non-dimensional expression is obtained from Equation (33):

$$
w^{2}=\frac{l^{2}}{4}\left(\frac{\psi}{\eta}+1+Z^{2}\right),
$$

where:

$$
\begin{aligned}
w & =\frac{\sigma^{0.5}}{H}, \\
l & =\left(\frac{\Omega}{R^{2}}\right)^{0.5}, \\
Z & =\frac{R}{H}, \\
Z^{2} & =f\left(l^{2}\right) .
\end{aligned}
$$

In Equation (38) $f\left(l^{2}\right)$ is, according to the pre-established considerations (see Equation (11)), a scale down function which takes into account the fact that that internal fluctuations will depend on the size of the microscopic entities and the size of the system.

Also, as there is a linear relation between the expected value of the radius and the perimeter, the non-dimensional variable $l$ is equivalent to the distance between two interface points. Consequently, the following scaling relation can be assumed:

$$
Z^{2}=f\left(l^{2}\right)=\left(1-\left(l^{2}\right)\right),
$$

So, Equation (34) is expressed as:

$$
w^{2}=\frac{l^{2}}{4}\left(\frac{\psi}{\eta}+2-l^{2}\right) .
$$

Substituting Equation (40) in (10) gives:

$$
\begin{aligned}
y & =\lim _{l \rightarrow 1} \frac{\Delta \ln \left(\left(\frac{l^{2}}{4}\left(\frac{\psi}{\eta}+2-l^{0.5}\right)\right)^{0.5}\right)}{\Delta \ln (l)} \\
& =\lim _{l \rightarrow 1}\left(\frac{\mathrm{d} \ln \left(\left(\frac{l^{2}}{4}\left(\frac{\psi}{\eta}+2-l^{0.5}\right)\right)^{0.5}\right)}{\mathrm{d} l}\right)\left(\frac{\mathrm{d} \ln (l)}{\mathrm{d} l}\right)^{-1} \\
& =\left(\frac{\psi}{\psi+\eta}\right) \\
y & =\left(\frac{\mu}{\mu+b}\right),
\end{aligned}
$$

And finally (41) in Equation (9) gives:

$$
d_{f}=2-\frac{1}{2}\left[C_{1}\left(\frac{\mu}{\mu+b}\right)+C_{2}\right],
$$

where the constants $C_{1}$ and $C_{2}$ are evaluated taking into account the interval of values physically possible that can be obtained by the relation between the reproduction and endogenous death rate constants. Then two extreme cases appear: 


$$
\frac{\psi}{\eta}=1 \Rightarrow d_{f}=2
$$

Because when $\psi / \eta=1$ the tumour does not grow so the fractal dimension is equal to the surface dimension, and:

$$
\frac{\psi}{\eta}=2 \Rightarrow d_{f}=1
$$

because when $\psi / \eta=2$ the contour rugosity is zero and the fractal dimension is equal to the topological dimension of the contour of a circle of radius $H$. Taking into account both extreme conditions given by Equations (43) and (44) the following expression is proposed to determine fractal dimension $d_{f}$ as a function of the quotient between mitosis and apoptosis rates [17], which quantifies the tumour capacity to invade and infiltrate healthy tissue [18].

$$
d_{f}=\left(\frac{5-\frac{\psi}{\eta}}{1+\frac{\psi}{\eta}}\right)
$$

\section{Results}

Diagnosis of tumour proliferation capacity and invasion capacity is very complex because these terms include many factors such as the tumour aggressiveness, which is related with the tumour growth rate $\dot{\xi}$, and the tumour invasion capacity, which is associated with the fractal dimension $d_{f}$ [18] among others factors.

In order to analyze the validity of the previously developed formalism, the tumour growth rate $(\dot{\xi}=\psi-\eta)$, where $\psi$ and $\eta\left[L . t^{-1}\right]$ are the macroscopic parameters associated to mitosis and apoptosis rate, macroscopically observed during the linear growth stage [9] was substi- tuted, for example, in Equation (7) resulting in:

$$
\dot{S}_{i} \approx R(\psi-\eta) \ln \frac{\psi}{\eta}
$$

The expression (46) represents the entropy production rate and includes the macroscopic parameters associated to mitosis and apoptosis rate $\psi$ and $\eta\left[L . t^{-1}\right]$ which are indexes which characterize tumour proliferation.

Substituting (45) in (46) the following formula is obtained:

$$
\dot{S}_{i}=R(\psi-\eta) \ln \left(\frac{5-d_{f}}{1+d_{f}}\right)
$$

In the formula (47) two properties observed in tumour growth are included. The first is its growth rate, which is associated with its invasive capacity $(\dot{\xi}=\psi-\eta)$. The second is its complexity, a morphology characteristic, such as the fractal dimension of the tumour interface, which quantifies the tumour capacity to invade and infiltrate the healthy tissue [18].

The entropy production rate (formula (XXXXVII)) was determined by fourteen tumour cell lines which are shown in Table 1. On one hand, as can be seen in cells lines with equal tumour invasion capacity, $d_{f}(\mathrm{Mv} 1 \mathrm{Lu}$ and AT5) but a different tumour aggressiveness, $\dot{\xi}$, exhibits differences on the entropy production rate. On the other hand, the cells lines with equal tumour aggressiveness, $\dot{\xi}$ but difference tumour invasion capacity, $d_{f}$ (HT-29 M6 and 3T3K-ras) exhibit differences taking place the entropy production rate. In other words, this unifying hallmark, allow us to use the entropy production rate as a physical function to measure cancer robustness.

In summary, this hallmark allow us, via the use of the entropy production rate to make a diagnosis of the tumor proliferation capacity and invasion capacity, key factors to improve cancer therapy.

Table 1. Entropy production rate for different tumour cell lines.

\begin{tabular}{cccc}
\hline Cell line & $d_{f}{ }^{(a)}$ & Growth rate $^{(\text {a) }} \dot{\xi}[\mu \mathrm{m} / \mathrm{h}]$ & Entropy production rate $\dot{S}_{i}[\mathrm{~J} \cdot \mu \mathrm{m} / \mathrm{mol} \cdot \mathrm{K} \cdot \mathrm{h}]$ \\
\hline Mv1Lu & 1.23 & 11.50 & 50.11 \\
AT5 & 1.23 & 8.72 & 38.06 \\
B16 & 1.13 & 5.83 & 30.08 \\
C-33a & 1.25 & 6.40 & 27.26 \\
VERO C & 1.18 & 5.10 & 23.77 \\
MCA3D & 1.09 & 3.73 & 19.45 \\
C6 & 1.21 & 2.90 & 13.46 \\
HT-29 & 1.13 & 1.93 & 9.64 \\
Car B & 1.20 & 2.06 & 9.39 \\
HT-29 M6 & 1.12 & 1.85 & 9.31 \\
3T3K-ras & 1.32 & 1.89 & 7.23 \\
HeLa & 1.30 & 1.34 & 5.32 \\
3T3 & 1.20 & 1.10 & 4.99 \\
Saos-2 & 1.34 & 0.94 & 3.49 \\
\hline
\end{tabular}

(a) Experimental results reported by Brú et al. [9] 


\section{Acknowledgements}

This work was support by a grant of the Higher Education Ministry of Cuba.

\section{References}

[1] T. S. Deisboeck, M. E. Berens, A. R. Kansal, S. Torquato, A. O. Stemmer-Rachamimov and E. A. Chiocca, "Pattern of Self-Organization in Tumour Systems: Complex Growth Dynamics in a Novel Brain Tumour Spheroid Model," Cell Proliferation, Vol. 34, 2001, pp. 115-134. doi:10.1046/j.1365-2184.2001.00202.x

[2] H. Kitano, "Towards a Theory of Biological Robustness," Molecular Systems Biology, Vol. 3, 2007, p. 137. doi:10.1038/msb4100179

[3] D. Rockmore, "Cancer Complex Nature," Santa Fe Institute Bulletin, Vol. 20, 2005, pp. 18-21.

[4] H. Kitano, "Cancer Robustness: Tumour Tactics," Nature, Vol. 426, 2003, p. 125.doi:10.1038/426125a

[5] H. Kitano, "Cancer as a Robust System: Implications for Anticancer Therapy," Nature Reviews Cancer, Vol. 4, 2004, pp. 227-235. doi:10.1038/nrc1300

[6] S. Hauptmann, "A Thermodynamic Interpretation of Malignancy: Do Genes Come Later?" Medical Hypotheses, Vol. 58, 2002, pp. 144-147.doi:10.1054/mehy.2001.1477

[7] L. F. Luo, "Entropy Production in a Cell and Reversal of Entropy Flow as an Anticancer Therapy," Frontiers of Physics in China, Vol. 4, 2009, pp. 122-136. doi:10.1007/s11467-009-0007-9

[8] J. M. Nieto-Villar, R. Quintana and J. Rieumont, "Entropy Production Rate as a Lyapunov Function in Chemical Systems: Proof," Physica Scripta, Vol. 68, 2003, pp. 163-165.

doi:10.1238/Physica.Regular.068a00163
[9] A. Brú, S. Albertos, J. L. Subiza, J. L. García-Asenjo and I. Brú, "The Universal Dynamics of Tumour Growth," Biophysical Journal, Vol. 85, 2003, pp. 2948-2961. doi:10.1016/S0006-3495(03)74715-8

[10] I. Prigogine, "Introduction to Thermodynamics of Irreversible Processes," Wiley, New York, 1961.

[11] Th. De Donder and P. Van Rysselberghe, "Thermodynamic Theory of Affinity, H. Milford," Oxford University Press, London, 1936.

[12] N. G. Van Kampen, "Stochastic Processes in Physics and Chemistry," 3rd Edition, Elsevier, Amsterdam, 2007.

[13] C. W. Gardiner, "Handbook of Stochastic Methods: For Physics, Chemistry and the Natural Sciences," 3rd Edition, Springer-Verlag, Heidelberg, 2004.

[14] V. Anishchenko, V. Astakhov, A. Neiman, T. Vadisova and L. Schimansky-Geir, "Nonlinear Dynamics of Chaotic and Stochastic Systems: Tutorial and Modern Developments," 2nd Edition, Springerr-Verlag, Heidelberg, 2007.

[15] V. A. Kuznetsov, I. A. Makalkin, M. A. Taylor and A. S. Perelson, "Nonlinear Dynamics of Immunogenic Tumours: Parameter Estimation and Global Bifurcation Analysis," Bulletin of Mathematical Biology, Vol. 56, 1994, pp. 295231.

[16] A. L. Barabasi and H. E. Stanley, "Fractal Concepts in Surface Growh,” Cambridge University Press, Cambridge, 1995. doi:10.1017/CBO9780511599798

[17] E. Izquierdo and J. M. Nieto-Villar, "Morphogenesis of the Tumour Patterns," Mathematical Biosciences and Engineering, Vol. 5, 2008, pp. 299-313.

[18] L. Norton, "Conceptual and Practical Implications of Breast Tissue Geometry: Toward a More Effective, Less Toxic Therapy," Oncologist, Vol. 10, 2005, pp. 370-381. doi:10.1634/theoncologist.10-6-370 